\title{
PRÉSTAMO DE LIBROS ELECTRÓNICOS EN BIBLIOTECAS PÚBLICAS. LA EXPERIENCIA DE EBIBLIO MADRID
}

\author{
Ebook lending services for public libraries. The \\ eBiblio Madrid experience
}

Remedios De-Vicente-García y Luisa-Inmaculada Fernández-Miedes
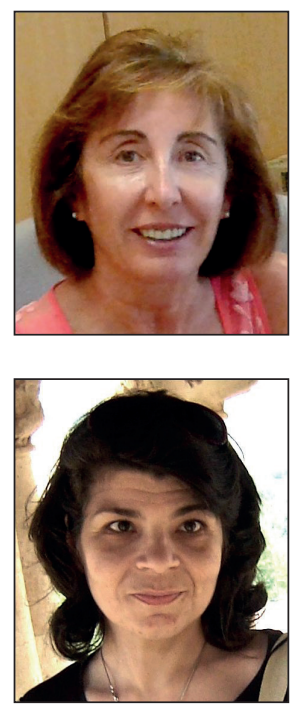

Remedios De-Vicente-García trabaja en la Unidad de Coordinación Técnica de la Subdirección General del Libro de la Comunidad de Madrid. Desarrolla su trabajo en tareas relacionadas con recursos electrónicos y desde 2014 se ocupa de la gestión de la plataforma de préstamo digital eBiblio Madrid. Coordina el grupo de trabajo de eBiblio Madrid y desde mayo de 2017 también es la coordinadora del grupo de trabajo de préstamo digital del Consejo de Cooperación Bibliotecaria, encargado de realizar el seguimiento y evaluación del servicio eBiblio.

https://orcid.org/0000-0002-1762-1183

remedios.vicente@madrid.org

Luisa-Inmaculada Fernández-Miedes trabajó en la Biblioteca de la Universidad Complutense de Madrid entre 2000 y 2006, y ese año ingresó como técnico superior facultativo de bibliotecas en la Comunidad de Madrid. Entre 2007 y 2011 dirigió la Biblioteca Regional de Madrid y desde ese año es responsable de la Unidad de Coordinación Técnica de la Subdirección General del Libro, unidad que lidera proyectos como eBiblio, el catálogo colectivo regional, estadística, equipamientos e implantación de nuevos servicios bibliotecarios transversales de alcance regional, especialmente los basados en herramientas tecnológicas.

https://orcid.org/0000-0003-4903-217X

Ifm10@madrid.org

Unidad de Coordinación Técnica, Subdirección General del Libro, Comunidad de Madrid Alcalá, 31. 28014 Madrid, España

\section{Resumen}

La puesta en marcha en septiembre de 2014 del proyecto de un servicio de préstamo de libros electrónicos, eBiblio, impulsado por el Ministerio de Educación, Cultura y Deporte en colaboración con las comunidades autónomas, inició una nueva etapa en las bibliotecas públicas españolas. El préstamo digital se incorporaba al resto de los servicios que ofrecían las bibliotecas y suponía hacer frente a numerosos retos para su gestión. Los cambios han afectado a los bibliotecarios, que han tenido que capacitarse tecnológicamente para utilizar un nuevo servicio, y a los usuarios que también necesitan adquirir una serie de habilidades tecnológicas para acceder a las lecturas. En este artículo se describen todos los elementos que confluyen en el préstamo digital y se analiza la evolución y las perspectivas de futuro del servicio, centrando algunos de estos aspectos en el caso concreto de eBiblio Madrid.

\section{Palabras clave}

Libro electrónico; Préstamo digital; Bibliotecas públicas; Colección; Plataformas tecnológicas; DRM; Difusión; eBiblio.

\section{Abstract}

With the launch in September 2014 of the project eBiblio to lend ebooks, promoted by the Spanish Ministry of Education, Culture and Sport in collaboration with the autonomous communities, began a new phase in the Spanish public libraries. The digital loan was incorporated into the rest of the services offered by the libraries and represented a number of management challenges. The changes have affected librarians who have had to be technologically trained to use a new service and users who also need to acquire a range of technological skills to access readings. In this article all aspects that converge in the digital loan will be described and the evolution and future prospects of the service will be analysed, focusing some of these aspects on the specific case of eBiblio Madrid.

\section{Keywords}

Ebook; Digital loans; Public libraries; Collections; Technological platforms; DRM; Dissemination; eBiblio. 
De-Vicente-García, Remedios; Fernández-Miedes, Luisa-Inmaculada (2018). "Préstamo de libros electrónicos en bibliotecas públicas. La experiencia de eBiblio Madrid". El profesional de la información, v. 27, n. 3, pp. 698-706.

https://doi.org/10.3145/epi.2018.may.23

\section{Introducción}

El proyecto eBiblio nació en 2013 cuando la Secretaría de Estado de Cultura licitó el contrato para implantar un sistema de gestión informática que permitiese la adquisición de licencias de uso de libros electrónicos para su préstamo en bibliotecas públicas (Cuadrado-Fernández; Fe-Trillo, 2015).

En septiembre de 2014 comenzó a funcionar progresivamente en todo el territorio español (excepto en el País Vasco donde dos meses más tarde se creó eLiburutegia, la plataforma de préstamo digital de la Red de Lectura Pública de Euskadi). En la Comunidad de Madrid la plataforma se denomina eBiblio Madrid, y está activa desde el 11 de septiembre de 2014.

Desde su inicio eBiblio cuenta con algunos rasgos distintivos respecto a otras experiencias similares:

- está implantado a nivel nacional, basado en la misma tecnología, estructura y concepto de servicio;

- se concibió con un carácter cooperativo: el Ministerio aportó la base tecnológica y los contenidos iniciales dando homogeneidad al proyecto, y los servicios autonómicos se ocupaban de la gestión efectiva;

- un aspecto diferenciador de eBiblio respecto a experiencias como la de las bibliotecas norteamericanas era la premisa de separar plataforma tecnológica y contenidos.

eBiblio surgió en un momento en el que la conexión a internet y la presencia de ordenadores y dispositivos móviles se encontraban muy extendidos entre la población. Según la Encuesta sobre equipamiento y uso de tecnologías de la información y la comunicación en los hogares en 2014 (INE, 2014), el porcentaje de viviendas con conexión a internet era de $74,4 \%$, con teléfono móvil un $96,4 \%$ y con ereaders un $20 \%$.

eBiblio cuenta con elementos que lo diferencian de otras experiencias similares: funciona a nivel nacional con carácter cooperativo, con la misma tecnología, estructura y concepto de servicio

También incidía en la necesidad y la oportunidad de un proyecto como eBiblio el Informe sobre piratería y hábitos de contenidos digitales en 2016 (GfK, 2017), que reflejaba que el $51 \%$ de los internautas accedía a contenidos de forma ilegal. El $15 \%$ de estas descargas eran de libros. En este contexto eBiblio se convertía en una alternativa legal a esta práctica.

La puesta en marcha de eBiblio Madrid supuso articular distintos elementos para lograr su funcionamiento:

- El principal problema de partida era el de la autenticación de usuarios: cuando se inició el servicio, la Comunidad de Madrid no disponía de un catálogo colectivo y no era posible a priori dar acceso a los usuarios de todas las bibliotecas de la región. Para conseguir este objetivo se implementó un formulario de preinscripción en el sistema de gestión bibliotecario a través de un modelo específico de opac (online public access catalog) y se otorgaron los permisos necesarios a los bibliotecarios para tramitar las altas de usuarios, limitadas al uso de eBiblio Madrid.

Se contaba con un equipo muy reducido que debía establecer mecanismos de coordinación a distintos niveles: con el Ministerio de Educación, Cultura y Deporte y el resto de comunidades autónomas a través del grupo de trabajo del Consejo de Cooperación Bibliotecaria, con los servicios bibliotecarios de la región, usuarios, editores y distribuidores.

El servicio partía sin asignación de presupuesto y progresivamente se ha ido dotando de recursos económicos para aumentar la colección, tareas de formación, difusión, etc.

\section{Elementos del servicio de préstamo digital}

\subsection{Plataformas tecnológicas}

Un aspecto clave en el préstamo digital es el papel que juegan las plataformas tecnológicas, en las que se definen todos los elementos relacionados con la gestión del servicio.

Libranda fue la empresa adjudicataria de la licitación del Ministerio de Educación, Cultura y Deporte hasta 2015. Tras un nuevo concurso, Odilo es la empresa adjudicataria hasta octubre de 2018.

Un aspecto clave en el préstamo digital es el papel de las plataformas tecnológicas, en las que se definen los elementos relacionados con la gestión del servicio

La experiencia en la gestión de la plataforma a lo largo de estos años ha servido para ir definiendo con más claridad las funciones necesarias para ofrecer un servicio óptimo.

Para facilitar al usuario la consulta y selección de los contenidos la interfaz debe tener una estructura clara y sencilla, organizada en categorías temáticas, sistemas de búsqueda simple y avanzada, y filtrado de resultados que permita la localización de contenidos con el menor ruido posible. Debe incluir ayudas y tutoriales, permitir la inclusión de banners informativos, opción de compartir en redes sociales, posibilidad de implementar clubes de lectura virtuales y de interactuar con el usuario.

España lidera el ranking mundial en penetración del uso de teléfonos inteligentes con un $81 \%$ de usuarios. Según la $20 \underline{a}$ Encuesta a usuarios de internet, navegantes en la Red, de la Asociación para la Investigación de Medios de Comunicación (AIMC, 2017), el 94,6\% de los encuestados se conecta 
diariamente a internet a través de su móvil. Es imprescindible pues que una plataforma de préstamo digital presente un diseño responsive para ofrecer una fácil navegación y visualización de contenidos en todo tipo de dispositivos.

El back office debe organizarse en diferentes áreas para gestionar el préstamo digital:

a) Gestión de la colección: con información estructurada para conocer en tiempo real el número de títulos y licencias, los datos asociados a cada título (fecha de adquisición, modalidad de compra, número de licencias, total de préstamos realizados en cada título, reservas y renovaciones) y alertas de caducidad de licencias.

\section{Las bibliotecas son un referente para el consumo legal de contenidos digitales al proteger los derechos de autor}

Incorporación de contenidos a través de catalogación directa o bien con importación de metadatos en formato marc, Onix.

b) Gestión de usuarios: número de inscritos, altas por períodos de tiempo, usuarios activos, usuarios por tipo de lector (adulto, infantil), género (hombre, mujer), rango de edad.

c) Datos estadísticos: número de visitas, páginas vistas, préstamos, reservas, renovaciones, descargas, visualizaciones en streaming, períodos de tiempo y tramos horarios, dispositivos utilizados (ereaders, dispositivos móviles, ordenador,) y porcentajes de lectura realizados.

d) Datos de uso de la colección desagregados por:

- tipo de documento (libros, revistas, periódicos, bases de datos, audiolibros, contenidos audiovisuales, recursos multimedia, etc.);

- formatos de lectura (epub, pdf, etc.);

- audiencia (adulto, infantil);

- autor;

- título;
- materias;

- uso por bibliotecas;

- grupos y sellos editoriales;

- ranking de obras más prestadas

e) Participación de usuarios: datos de obras más valoradas y comentadas.

Una plataforma de préstamo digital debe disponer de una aplicación para dispositivos móviles que permita el acceso al catálogo por categorías de materias, búsqueda simple o avanzada, información de la cuenta del usuario, y contar con funciones asociadas al préstamo (préstamo, devolución, renovación) y a la lectura (opciones de tamaño y tipo de letra, interlineado, brillo, color de fondo, subrayado, marcadores, notas, búsqueda en diccionario).

En el diseño y configuración de la aplicación deben intervenir elementos para que la experiencia de usuario sea lo más amigable posible:

- navegación fácil para localizar y acceder a los contenidos de manera rápida y sencilla;

- opciones de compartir en redes sociales, establecer listas de lecturas;

- participación a través de comentarios sobre las obras y valoraciones mediante puntuación, estrellas, etc.

\subsection{DRM (digital rights management)}

La preservación de los derechos de autor y los sistemas de protección de éstos mediante DRM, que establece los usos permitidos por el titular de los derechos sobre una obra digital, es uno de los aspectos más controvertidos del libro electrónico (Alonso-Arévalo, 2015).

Las bibliotecas son un referente para el consumo legal de contenidos digitales al proteger los derechos de autor. No obstante, el uso de DRM supone algunos problemas al usuario para el préstamo y la lectura de las obras.

Las operaciones necesarias para la instalación del programa Adobe digital editions, obtención del ID, autorización de los dispositivos y transferencia al ereader son un obstáculo

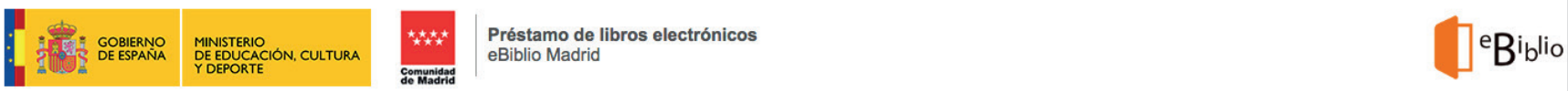

\begin{tabular}{|l|l|l|l|l|}
\hline Inicio & Avanzada \\
Obras de consulta, \\
vídeos, \\
documentales y \\
musica
\end{tabular}

Figura 1. Sitio web eBiblio Madrid. Recursos en streaming

http://www.madrid.ebiblio.es 


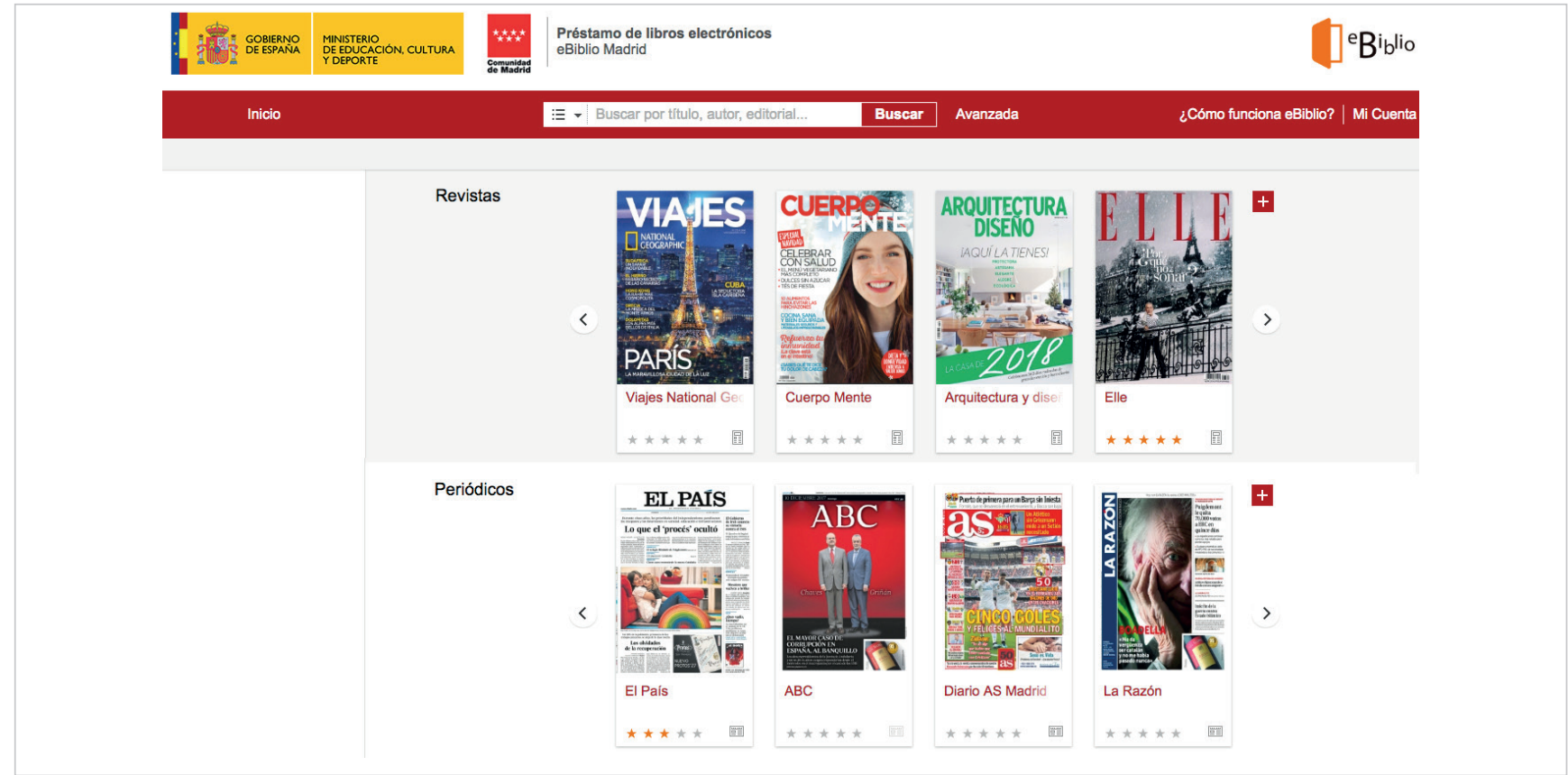

Figura 2. Sitio web eBiblio Madrid. Periódicos y revistas http://www.madrid.ebiblio.es

para efectuar el préstamo. La incompatibilidad de formatos y DRM propietarios, como el DRM de Amazon, impide a un elevado número de usuarios que disponen de ereader Kindle, utilizar eBiblio.

\subsection{Colección digital. Modelos en la adquisición de los contenidos}

La gestión de la propiedad intelectual de las obras digitales es más compleja que la del libro impreso. La distribución de contenido por las redes informáticas es un acto de comunicación pública, se considera un servicio y se regula de manera diferente a la mera distribución.

Los documentos se adquieren a través de suscripciones o licencias que fijan las condiciones de acceso. Los libros están sujetos a los usos autorizados establecidos en los contratos.

En eBiblio la adquisición de licencias de uso de libros electrónicos se licitó en el contrato del Ministerio de Educación, Cultura y Deporte: un lote inicial para todas las comunidades autónomas de 1.190 títulos de libros electrónicos y audiolibros, a los que se sumarían lotes de novedades con carácter trimestral (Cuadrado-Fernández; Fe-Trillo, 2015).

Las licencias de los títulos tenían una duración de dos años. En el último contrato de licitación (2017), la modalidad de compra se modificó y los títulos se adquirieron para un uso mínimo de 25 préstamos en cada licencia.

La Comunidad de Madrid incrementó en 2015 la colección con 632 títulos y 2.265 licencias (con un coste de $17.496,48 €$ ). Durante 2016 adquirió 963 títulos y 7.065 licencias por valor de $61.683,82 €$.

En 2017 además de ebooks se adquirieron audiolibros, libros enriquecidos (texto y audio), periódicos y contenidos en streaming (obras de referencia, vídeos y documentales, música). En total 2.843 títulos y 13.849 licencias por un importe de $176.321,16 €$.

En la suscripción por licencias, la experiencia de eBiblio ha reportado información a analizar y tener en cuenta para la gestión de las colecciones de préstamo digital.

La suscripción de contenidos por un período temporal supone que todos los libros adquiridos en la misma fecha, independientemente de su uso y número de préstamos, caducan transcurrido el período de suscripción. El resultado es un descenso brusco de títulos y la necesidad de renovar y adquirir de nuevo las obras para mantener un catálogo con un crecimiento sostenido a lo largo de los años.

Tras esta experiencia las bibliotecas plantearon a editoriales y distribuidores la necesidad de buscar soluciones más flexibles para la adquisición de contenidos que contemplaran la opción de suscripción por número de préstamos, fijando un mínimo de 25 préstamos por licencia.

Esta opción, aunque en general beneficia al grueso de la colección, también plantea algunos problemas en los títulos más prestados de best sellers y novedades, que cuentan con un elevado número de préstamos concentrados en poco tiempo. En estos casos las licencias por número de préstamos se agotan con rapidez y al renovarlas se adquieren al mismo precio que en su primera compra, sin ningún descuento adicional.

Es imprescindible explorar modelos de adquisición, buscando fórmulas que incluyan tipos de licencias según el ciclo de vida del libro (fondo o novedad), género de las obras, adquisición para clubes de lectura mediante concurrencia, etc.

En el futuro se puede proponer para las bibliotecas públicas la adquisición consorciada de obras y recursos digitales. Esta 
Tabla 1. Datos de registro de altas de usuarios en eBiblio Madrid 2015-2017

\begin{tabular}{|l|r|r|r|}
\hline \multicolumn{1}{|c|}{ N. altas usuarios } & $\mathbf{2 0 1 5}$ & $\mathbf{2 0 1 6}$ & $\mathbf{2 0 1 7}$ \\
\hline Enero & 1.379 & 604 & 777 \\
\hline Febrero & 1.054 & 503 & 669 \\
\hline Marzo & 820 & 580 & 647 \\
\hline Abril & 598 & 474 & 545 \\
\hline Mayo & 577 & 368 & 486 \\
\hline Junio & 610 & 386 & 494 \\
\hline Julio & 555 & 470 & 585 \\
\hline Agosto & 563 & 501 & 594 \\
\hline Septiembre & 607 & 622 & 747 \\
\hline Octubre & 600 & 660 & 776 \\
\hline Noviembre & 645 & 666 & 1.210 \\
\hline Diciembre & 474 & 584 & 1.164 \\
\hline Total & 8.482 & 6.418 & 8.702 \\
\hline
\end{tabular}

fórmula, de manera similar a lo que hacen las bibliotecas universitarias con las revistas académicas, facilitaría negociar unas condiciones de compra de licencias más ventajosas.

Es imprescindible explorar modelos de adquisición, buscando fórmulas que incluyan tipos de licencias según el ciclo de vida del libro

eBiblio Madrid tiene también como objetivo diversificar su oferta. Durante 2017 se han incluido nuevos recursos para su consumo en streaming:

- obras de referencia y consulta: Mienciclo hispánica universal, Aula digital y Patrimonio de la humanidad: http://www.grupoenciclo.com/recursos_electronicos.html

- acceso a la base de datos de Alexander Street en inglés, con más de 44.000 vídeos que ofrece documentales de viajes, arte, psicología, historia, audio, óperas, música y partituras.

En octubre de 2017 se incluyeron cinco periódicos: $A B C, A S$, Cinco días, La razón y El país, que suscitaron el interés de los usuarios tal y como demuestran las cifras de acceso ${ }^{1}$. En octubre los periódicos supusieron un $14 \%$ del total de los préstamos realizados en ese mes, en noviembre un $23 \%$ y en diciembre un $19 \%$.

En noviembre de 2017 el Ministerio de Educación, Cultura y Deporte suscribió para todas las comunidades autónomas una selección de 31 revistas de temas diversos (gastronomía, moda, viajes, historia, literatura, medicina y salud, etc.). En diciembre el porcentaje de préstamos de las revistas fue del $19 \%$ sobre el total de los préstamos en eBiblio Madrid.
Tabla 2. Número mensual de usuarios activos en eBiblio Madrid 2015-2017

\begin{tabular}{|l|l|l|l|}
\hline \multicolumn{1}{|c|}{ Usuarios activos } & $\mathbf{2 0 1 5}$ & $\mathbf{2 0 1 6}$ & $\mathbf{2 0 1 7}$ \\
\hline Enero & 2.326 & 2.664 & 3.646 \\
\hline Febrero & 2.221 & 2.502 & 3.503 \\
\hline Marzo & 2.190 & 2.749 & 3.560 \\
\hline Abril & 1.978 & 2.677 & 3.398 \\
\hline Mayo & 1.904 & 2.461 & 3.437 \\
\hline Junio & 1.974 & 2.572 & 3.376 \\
\hline Julio & 1.905 & 2.681 & 3.730 \\
\hline Agosto & 1.983 & 2.590 & 3.946 \\
\hline Septiembre & 2.190 & 2.825 & 4.218 \\
\hline Octubre & 2.664 & 2.918 & 4.450 \\
\hline Noviembre & 2.513 & 3.136 & 5.198 \\
\hline Diciembre & 2.386 & 3.257 & 5.433 \\
\hline
\end{tabular}

\section{Evolución de eBiblio Madrid}

\subsection{Altas de usuarios registrados}

En 2015 se produjo un número elevado de altas de usuarios, reduciéndose durante 2016 con un $-24 \%$ de usuarios registrados (en parte debido a los problemas derivados del cambio de plataforma tecnológica). Esta tendencia negativa se invierte en 2017 , con un incremento en el número de altas de lectores de un $36 \%$.

\subsection{Usuarios activos}

El número de prestatarios activos durante 2017 fue de 12.923, un 39\% más que en 2016 (9.288).

\subsection{Préstamos}

El número de préstamos muestra una evolución positiva durante 2016 y 2017, con un aumento del $48 \%$ en 2016 y un $63 \%$ en 2017. El número de préstamos por 1.000 habitantes en 2016 fue de 14,2 y en 2017 de 22,8.

El incremento de la colección influye de manera directa en el ascenso de los préstamos. Los meses con mayor número

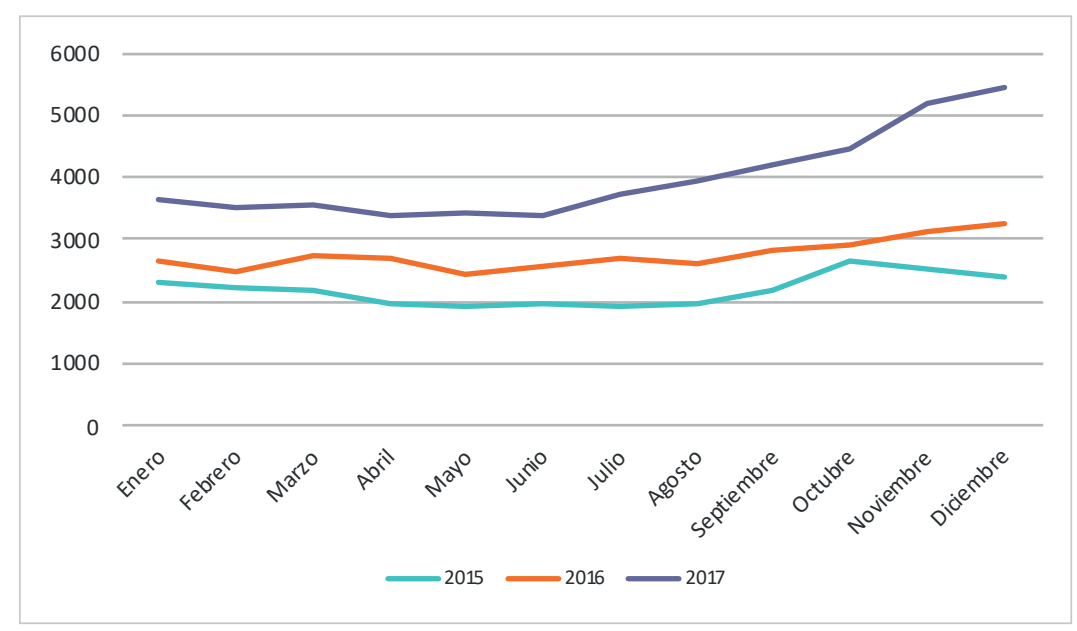

Gráfico 1. Evolución mensual del número de usuarios activos en eBiblio Madrid 2015-2017 
de préstamos coinciden con los meses en que se realizaron más adquisiciones en eBiblio Madrid (octubre de 2015, de octubre a diciembre en 2016, marzo de 2017, y de julio a diciembre de 2017). Otro factor fundamental en el incremento de las cifras de préstamo a partir de octubre fue la incorporación de periódicos y revistas.

\subsection{Materias más prestadas}

Los títulos más prestados han sido los de ficción en sus distintos géneros. Durante 2016 el $61 \%$ de los préstamos fueron obras de ficción, un $5 \%$ infantil y juvenil, 9\% de desarroIlo personal y autoayuda y el $25 \%$ restante en otras materias. En 2017 la ficción representó el 65\% de los préstamos, obras de humanidades e infantil y juvenil $5 \%$ respectivamente, desarrollo personal y autoayuda el $6 \%$, audiolibros un $4 \%$ y el $15 \%$ restante otras materias.

\section{La biblioteca, pieza fundamental para el desarrollo de servicios de lectura digital}

Las bibliotecas son un referente para la consolidación de la lectura digital, y lideran proyectos que favorecen la inclusión y la democratización de la cultura desde el formato digital.

A menudo se ha entendido la biblioteca digital como una nue-
Tabla 3. Número de préstamos en eBiblio Madrid 2015-2017

\begin{tabular}{|l|c|c|r|}
\hline \multicolumn{1}{|c|}{ N. de préstamos } & $\mathbf{2 0 1 5}$ & $\mathbf{2 0 1 6}$ & $\mathbf{2 0 1 7}$ \\
\hline Enero & 5.041 & 7.136 & 10.064 \\
\hline Febrero & 4.721 & 6.770 & 8.679 \\
\hline Marzo & 5.078 & 7.644 & 9.208 \\
\hline Abril & 4.597 & 7.690 & 8.798 \\
\hline Mayo & 4.416 & 6.919 & 9.347 \\
\hline Junio & 4.590 & 7.327 & 9.570 \\
\hline Julio & 4.781 & 7.578 & 10.898 \\
\hline Agosto & 5.270 & 7.441 & 11.595 \\
\hline Septiembre & 5.358 & 7.392 & 12.948 \\
\hline Octubre & 5.991 & 7.730 & 14.001 \\
\hline Noviembre & 6.076 & 8.551 & 20.659 \\
\hline Diciembre & 5.676 & 8.959 & 23.103 \\
\hline Total & 61.595 & 91.137 & 148.870 \\
\hline
\end{tabular}

va forma de biblioteca en sí misma. En esta visión reemplaza a la biblioteca física. Esta es una de las razones que ha llevado a una parte de los bibliotecarios a entender los contenidos digitales como una competencia y una amenaza. El desconocimiento y el desconcierto que suscitan los nuevos contenidos, un cierto sentimiento de imposición y el esfuerzo requerido para adquirir las competencias necesarias han provocado en algunos casos que las bibliotecas se desentendieran de las oportunidades que estos servicios suponían.

Un proyecto como eBiblio reafirma los elementos bibliotecarios. Basa en pautas técnicas los criterios de selección y preserva la intimidad de los datos de los lectores, diferenciándose de los servicios de plataformas comerciales.

Los usuarios que han dejado de acudir a las bibliotecas son potenciales lectores interesados en una alternativa que se ajuste más a sus necesidades. Aproximadamente un $20 \%$ de usuarios inscritos en eBiblio Madrid en estos tres años son lectores que utilizaron en el pasado el servicio de préstamo convencional y que hacía más de cinco años que no lo utilizaban.

El catálogo online es el eje de la gestión de la biblioteca y la información de los contenidos digitales debe incluirse en el sistema de gestión. Actualmente sólo se ha conseguido un cierto nivel de integración con la base de datos de usuarios para tareas de autenticación.

Gráfico 3. Préstamo por materias en eBiblio Madrid 2017 
Esta necesaria convergencia plantea dificultades técnicas como la agregación y normalización de la información bibliográfica en el catálogo. Para ello se ha de trabajar en varias líneas:

- normalización de metadatos desde las plataformas editoriales y de venta;

- agregación y recolección de contenidos entre plataformas;

- tratamiento de estos recursos en la evolución de los sistemas de gestión de bibliotecas en España dentro de la implantación de los estándares previstos en los próximos años.

La integración total con el catálogo presenta dificultades derivadas de la variedad de recursos digitales, de su forma de contratación, gestión y acceso.

El mantenimiento del catálogo de las colecciones en papel se basa en información unívoca de ejemplares identificables a través del alta y la baja previa intervención humana. La consistencia de la información que presta el catálogo en las colecciones digitales debe tener en cuenta la gestión de nuevos conceptos como la caducidad y la convivencia de distintos modelos de adquisición.

Un 20\% de usuarios inscritos en eBiblio Madrid son lectores que utilizaron en el pasado el servicio de préstamo convencional y que hacía más de 5 años que no lo utilizaban

\section{Fortalezas de eBiblio}

El préstamo de contenidos digitales permite:

- Llegar a todas las personas: un servicio como eBiblio se convierte en el mejor servicio de extensión bibliotecaria al ofrecer acceso durante 24 horas y 365 días.

- Fomentar la cooperación bibliotecaria: eBiblio es un servicio cooperativo a nivel nacional y regional que aúna los esfuerzos de profesionales implicados en mejorar la calidad del servicio optimizando los recursos disponibles (Fernández-Miedes; De-Vicente-García, 2015).

- Capacitar a los usuarios en el uso de nuevas tecnologías: las tareas de alfabetización digital que se realizan en la biblioteca contribuyen a reforzar el servicio de eBiblio.

- Respetar los derechos de propiedad intelectual: eBiblio fomenta el consumo legal de contenidos remunerados para los autores y el resto de agentes de la cadena del libro y hace compatible el uso de la obra por el lector (notas, comentarios), el enriquecimiento del texto (diccionario, enlaces, contenidos hipermedia) con la integridad de la obra (Fernández-Miedes; De-Vicente-García, 2015).

- Aportar variedad de oferta y selección de fondos con criterios profesionales: los estudios de usuarios de bibliotecas concluyen que la selección realizada por los bibliotecarios se valora positivamente. La biblioteca no se ve sometida a la presión del mercado y de los resultados de las ventas. En el ecosistema digital el rol del mediador es fundamental para localizar y difundir contenidos de calidad adecua- dos a cada lector, más allá de los algoritmos de las grandes empresas distribuidoras.

- Favorecer la visibilidad de la colección: las colecciones digitales facilitan el descubrimiento de nuevos contenidos a los usuarios. La presentación de los recursos se realiza con un acceso sencillo a obras y temas (novedades, recomendaciones, más prestados, revistas y periódicos, selecciones temáticas, etc.), para situar las colecciones digitales dentro del concepto de colección por centros de interés. Las estanterías virtuales se configuran en función de puntos de vista o contenidos dinámicos a través de las tareas de curación del fondo para favorecer la experiencia del usuario.

- Salvaguardar la intimidad y protección de datos de los usuarios; las instituciones están comprometidas en el respeto de los datos de los usuarios para usos exclusivamente relacionados con la prestación de los servicios.

La presentación de los recursos en estanterías virtuales sitúa a las colecciones digitales dentro del concepto de colección por centros de interés

\section{Puntos débiles}

1) Dificultades tecnológicas, incompatibilidad de formatos, DRM: la utilización de DRM y los problemas e incidencias que se producen con el programa Adobe digital editions dificultan y disuaden a los usuarios con menos habilidades tecnológicas para utilizar el préstamo digital.

Los sistemas de préstamo deben ser sencillos y fáciles de usar. Según los datos del Observatorio de la piratería y hábitos de consumo de contenidos digitales en 2016 (GfK, 2017), uno de los motivos de los usuarios que descargan contenidos de manera ilegal es la rapidez y facilidad de uso (42\%). Los sistemas de protección de derechos de autor son indispensables para los contenidos digitales, pero se debería evolucionar a sistemas de DRM que permitan la gestión de los derechos electrónicos con seguridad y faciliten la experiencia de lectura.

2) Escasa difusión y visibilidad: existe un desconocimiento de eBiblio por la mayoría de la población.

Se deben emprender acciones encaminadas a llegar al mayor número de ciudadanos y realizar campañas en medios de comunicación, redes sociales, bibliotecas, centros culturales, instituciones educativas, lugares de ocio, centros de salud, establecer colaboraciones con influencers, booktubers, etc.

La Subdirección de Cooperación Bibliotecaria y las comunidades autónomas tienen previsto establecer nuevas estrategias conjuntas de difusión de eBiblio. Una de las primeras medidas fue la realización en enero de 2018 de tareas de difusión en redes sociales, Google y una campaña bajo el lema "Hazte socio".

Es indispensable que desde cada comunidad autónoma se planifiquen estrategias de difusión. En el caso de la Comu- 
nidad de Madrid se han distribuido folletos, carteles y bolsas en los servicios de lectura de la región, se han colocado adhesivos en los bibliobuses con el logo de eBiblio, algunas bibliotecas de la región difunden el servicio en sus redes sociales y se incluye información sobre eBiblio Madrid de manera regular en Canal Metro.

En la Comunidad de Madrid, el reciente Estudio de hábitos de lectura y compra de libros en la Comunidad de Madrid 2016 (Comunidad de Madrid, 2017) refleja que un 36\% de los lectores lee habitualmente en el transporte público en su tiempo libre e invierte una media de 41 minutos en la lectura; el metro con un $72,5 \%$ de usuarios es el medio de transporte donde más se lee.

Emprender campañas de difusión enfocadas directamente al transporte público contribuiría a que eBiblio fuera más conocida por los usuarios.

Recientemente Google ha implementado en Estados Unidos la función Google Search como parte de la tarjeta Knowledge Graph Card, que ayuda a dar visibilidad a los libros electrónicos en plataformas de préstamo digital en las bibliotecas. Al buscar un libro, muestra los sistemas de biblioteca pública cercanos a la ubicación de la persona que realiza la búsqueda y proporciona un enlace para abrir la web y pedir el libro electrónico en préstamo (Alonso-Arévalo, 2017). Si esta función se incorporara en España, influiría de manera muy positiva en la visibilización de eBiblio y en el aumento del número de usuarios, si bien el uso de los datos de los usuarios y su explotación comercial es un factor a sopesar en este tipo de iniciativas.

3) Colección insuficiente: aún no se ha alcanzado un número de títulos que permita asentar el servicio de préstamo digital. El número de títulos ${ }^{2}$ oscila entre comunidades que están por debajo de los 2.000 títulos y otras como Cataluña (7.194 títulos) y Madrid (4.565 títulos), que tienen el mayor número de recursos en eBiblio.

Hasta que no se alcancen unos 10.000 títulos no se puede considerar que un servicio de préstamo digital pueda dar cobertura mínima y suficiente a los usuarios.

4) Escasa presencia de usuarios infantiles y juveniles, según reflejan los datos estadísticos; en eBiblio Madrid sólo se utiliza un $5 \%$ de la colección infantil y juvenil, y será necesario plantear acciones concretas para atraer a este segmento de usuarios que presentan condiciones específicas de acceso.

\section{Conclusiones}

La biblioteca debe defender el interés público en el nuevo contexto de la información y para ello precisa entender y conocer los nuevos modelos digitales en los que se crea, accede y difunde la información en el siglo XXI (Pérez-Adsuar-Belso, 2017).

El proyecto eBiblio se va afianzando paulatinamente y cuenta con una sólida base sobre la que evolucionar. Permitirá estudiar distintos contenidos y experimentar otras formas de lectura y comunicación con el usuario, adaptándose a las nuevas modalidades de consumo de contenidos culturales.

En los próximos años se deberá trabajar en varias líneas para consolidar eBiblio:
- Mantener una continuidad de la plataforma (ya sea ligada al proyecto del Ministerio de Educación, Cultura y Deporte, o bien de manera independiente según evolucione el proyecto) con mejoras continuas en los desarrollos tecnológicos para la gestión y prestación del servicio.

- Incluir todo tipo de recursos, más allá de los ebooks, y vincular los contenidos con las actividades de las bibliotecas.

- Realizar estudios de usuarios y detectar sus necesidades, tendencias y preferencias de consumo.

En el marco de la evolución de los modelos de negocio, las bibliotecas deberán trabajar activamente con los editores para encontrar soluciones beneficiosas para todos los agentes implicados en el sector del libro y analizar modelos más eficientes para alcanzar una sostenibilidad razonable a medio y largo plazo.

Las bibliotecas serán agentes activos en la defensa de los derechos de los lectores digitales, tendrán que impulsar nuevas formas de acceso a los contenidos y fomentar sistemas de protección de los contenidos digitales más amigables y avanzados, que permitan superar las limitaciones de los DRMs actuales.

Una de las líneas de actuación para consolidar eBiblio será realizar estudios de usuarios y detectar sus necesidades, tendencias y preferencias de consumo

Una nueva forma de leer y de acceder al conocimiento se abre paso y coexistirá junto a los hábitos tradicionales. Como defendía Neil Galman en su conferencia para la Reading Agency, "las bibliotecas son las puertas del futuro", son el lugar en el que se encuentra información, se adquieren competencias y el pasado, el presente y el futuro se dan la mano (Gelman, 2013).

\section{Notas}

1. Períodos de préstamo. Periódicos, 2 horas; revistas, 24 horas; ebooks, 21 días.

\section{Datos extraídos a 2 de enero de 2018.}

\section{Referencias}

AIMC (2017). 20 Encuesta navegantes en la Red. Asociación para la Investigación de Medios de Comunicación. http://download.aimc.es/aimc/REP2a3z/macro2016.pdf

Alonso-Arévalo, Julio (2015). “¿Qué son y para qué sirven los DRM? Universo abierto, 22 diciembre. https://universoabierto.org/2015/12/22/que-son-y-paraque-sirven-los-drm

Alonso-Arévalo, Julio (2017). “Google permite visualizar en sus búsquedas los libros electrónicos disponibles en sus bibliotecas". Universo abierto, 21 septiembre.

https://universoabierto.org/2017/09/21/google-permitevisualizar-en-sus-busquedas-los-libros-electronicosdisponibles-en-prestamo-en-bibliotecas

Comunidad de Madrid (2017). Estudios de hábitos de lectura y compra de libros en la Comunidad de Madrid 2016. 
https://goo.gl/iA59L2

Cuadrado-Fernández, María-Isabel; Fe-Trillo, María-José (2015). "eBiblio, servicio de préstamo de libros electrónicos en bibliotecas públicas". El profesional de la información, v. 24, n. 2, pp. 176-184.

https://doi.org/10.3145/epi.2015.mar.11

Fernández-Miedes, Luisa-Inmaculada; De-Vicente-García, Remedios (2015). "Bibliotecas centenarias. Libro electrónico y nuevas formas de lectura". En: Leyendo Madrid. Cien años de bibliotecas públicas. Madrid: Comunidad de Madrid, pp. 83-96. https://goo.gl/hANsZ6

Gaiman, Neil (2013). "Por qué nuestro futuro depende de las bibliotecas, de la lectura y de soñar despiertos". Fundación Asimov, 14 julio.

https://goo.gl/hzox4X
GfK (2017). Observatorio de piratería y hábitos de consumo de contenidos digitales 2016.

http://lacoalicion.es/wp-content/uploads/observatoriopirateria-y-habitos-consumo-2016.-ejecutivo.es_.pdf

INE (2014). Nota de prensa. Encuesta sobre equipamiento y uso de tecnologías de información y comunicación en los hogares. Año 2014. Instituto Nacional de Estadística.

http://www.ine.es/prensa/np864.pdf

Ministerio de Educación, Cultura y Deporte (2017). Ebiblio: préstamo de libros electrónicos en bibliotecas públicas.

https://www.mecd.gob.es/cultura/areas/bibliotecas/mc/ eBiblio/estadisticas.html

Pérez-Adsuar-Belso, Antonio (2017). La odisea del libro. La transición digital. Guía para autores, editores, libreros y bibliotecarios. Barcelona: Diéresis. ISBN: 9788494628917

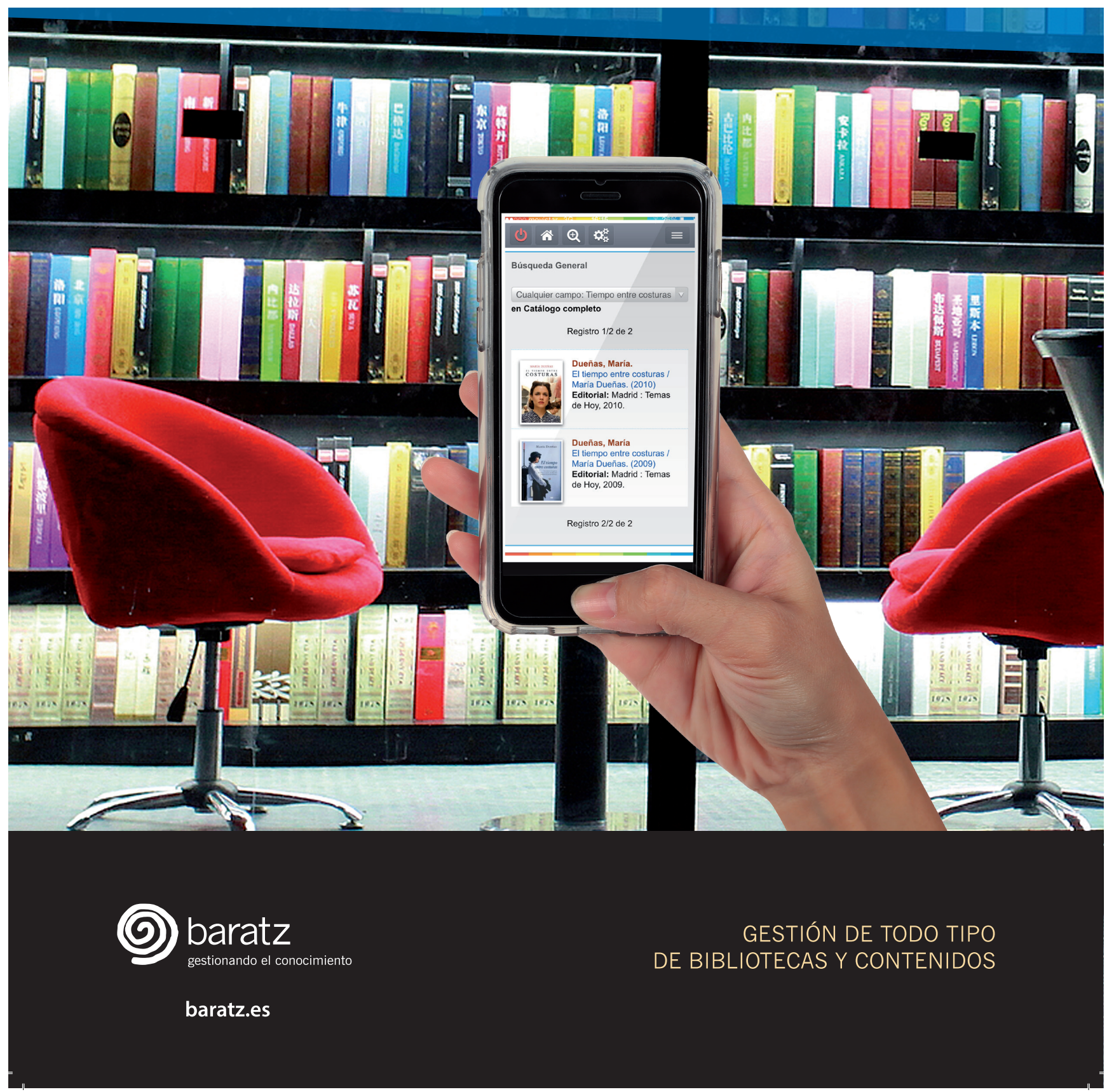

